\title{
Perceived and Actual Knowledge of Over the Counter Anti-inflammatory Medications Among Athletic Trainers
}

ISSN: 2577-1914

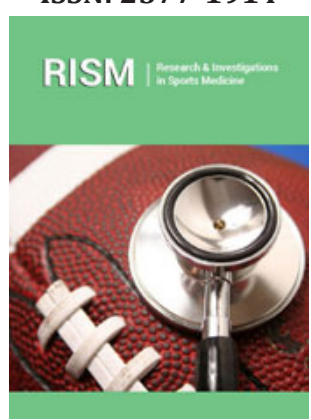

*Corresponding author: S Andrew C, University of Texas at Tyler, USA

\section{Submission: 非 August 12, 2020}

Published: 毕September 22, 2020

Volume 7 - Issue 1

How to cite this article: $S$ Andrew $C$, Brandon W, Diana G, David M, Marc E. Perceived and Actual Knowledge of Over the Counter Anti-inflammatory Medications Among Athletic Trainers. Res Inves Sports Med, 7(1): RISM.000654. 2020. DOI: $10.31031 /$ RISM.2020.07.000654

Copyright@ S Andrew C, This article is distributed under the terms of the Creative Commons Attribution 4.0 International License, which permits unrestricted use and redistribution provided that the original author and source are credited.

\author{
S Andrew $\mathrm{C}^{1,2 *}$, Brandon $\mathbf{W}^{2,3}$, Diana $\mathrm{G}^{1}$, David $\mathbf{M}^{3}$ and Marc $\mathrm{E}^{3}$ \\ ${ }^{1}$ University of Texas at Tyler, USA \\ ${ }^{2}$ University of North Carolina, Greensboro, USA \\ ${ }^{3}$ Grand Canyon University, USA
}

\begin{abstract}
Recent studies have suggested a gap between perceived and actual knowledge of different therapeutic modalities among athletic trainers. However, the authors of these studies have noted that further investigation into athletic training populations is needed to determine the full extent of knowledge gaps related to athletic training skills and tasks. To the authors' knowledge, there have been no studies conducted to describe the perceived and actual knowledge of over the counter anti-inflammatory medications among athletic trainers. Thus, the purpose of this study was to describe the perceived and actual knowledge of over the counter anti-inflammatory medications among athletic trainers. A total of 96 athletic trainers participated in this study (age $=43 \pm 13$ years, certified experience $=19 \pm 12$ years). Participants were sent an electronic survey by email that assessed frequency of usage, perceived knowledge, and actual knowledge of cupping therapy. Measures of central tendency (means, standard deviations, frequencies) were calculated for all survey items. Data was downloaded and analyzed using a commercially available statistics package (SPSS Version 26, IBM, Armonk, NY). A Pearson correlation was calculated for the perceived knowledge mean score and the actual knowledge total score to identify a knowledge gap between what participants believed they knew and what they actually knew. The majority of participants also reported using over the counter anti-inflammatory medications during a typical week of clinical practice. Regarding perceived knowledge, the majority of participants were above the midrange of agreement/disagreement, indicating at least some confidence about their knowledge of over the counter anti-inflammatory medications. Average scores on actual knowledge were $7.40 \pm 3.12$. A weak positive relationship was found between perceived knowledge and actual knowledge of over the counter anti-inflammatory medications $(\mathrm{r}=0.241, \mathrm{P}=0.018)$. As with previous studies on athletic training skills and knowledge, the average scores for actual knowledge were lower than $75 \%$. This demonstrates a need for improved knowledge transfer and retention with regards to over the counter anti-inflammatory medications used by athletic trainers. Future research should assess perceived and actual knowledge of other athletic training knowledge and skills, and the best practices for improving knowledge transfer and retention.
\end{abstract}

\section{Introduction}

Non-steroidal anti-inflammatory drugs (NSAIDs) have been recommended for reducing the inflammatory process, decreasing pain, and decreasing fever [1-4]. By acting on various chemical receptor sites, these medications inhibit two isoforms of cyclooxygenase, specifically COX-1 and COX-2 [1,2]. By inhibiting COX-2, NSAIDs reduce inflammation, pain, and fever $[1,2]$. According to previous studies, NSAIDs are used regularly by high performance athletes $[3,4]$. Additionally, over the counter NSAIDs are commonly administered by athletic trainers during their clinical practice $[5,6]$. While previous studies have been published suggesting that athletic trainers are uncomfortable with their knowledge of opioid medications, there do not appear to be any studies published on the perceived and actual knowledge of over the counter NSAIDs among athletic trainers [7].

Considering this lack of research on athletic trainers' knowledge of over the counter NSAIDs, the widespread use of the medications among athletes may present potential issues. Previous research suggests that athletes in some sports may be using NSAIDs incorrectly, or not using them for their intended use [6,8]. Furthermore, NCAA Division I football players reported getting over a quarter of the over the counter NSAIDs they ingested from their athletic trainer [6]. Previously surveyed athletic trainers felt they had a role in the use of 
medications with their student-athletes [7]. If this is the case, it is important to ensure that athletic trainers have an appropriate level of knowledge regarding the medications they are administering. Therefore, the purpose of this study was to determine the perceived and actual knowledge of over the counter NSAIDs among athletic trainers.

\section{Methods}

\section{Design}

This study was conducted using a cross-sectional design utilizing an internet-based survey for data collection.

\section{Participants}

Participants were recruited for this study by emailing head athletic trainers at colleges and universities in the United States, program directors for accredited athletic training programs, and athletic trainers in local professional organizations. A total of 96 participants (age $=43 \pm 13$ years, certified experience $=19$ \pm 12 years) opened the survey link and completed the survey in its entirety. Demographic information about the participants is presented in Table 1. All participants were informed of the survey's purpose as part of the beginning of the survey, at which point informed consent was obtained.

Table 1: Totals and percentage for participant demographic information.

\begin{tabular}{|c|c|c|}
\hline Demographic Factor & Criteria & Responses \\
\hline \multirow{2}{*}{ Sex } & Male & $51,53.1 \%$ \\
\hline & Female & $45,46.9 \%$ \\
\hline \multirow{4}{*}{ Clinical Practice Setting } & College/University & $74,77.1 \%$ \\
\hline & Secondary School & $17,17.7 \%$ \\
\hline & Clinic/Hospital & $4,4.2 \%$ \\
\hline & Emerging Settings & $1,1.0 \%$ \\
\hline \multirow{6}{*}{ Highest Degree Earned } & Professional Bachelor's & $9,9.4 \%$ \\
\hline & Professional Master's & $10,10.4 \%$ \\
\hline & Post-Professional Masters (in Athletic Training) & $17,17.7 \%$ \\
\hline & Post-Professional Master's (not in Athletic Training) & $38,39.6 \%$ \\
\hline & Post-Professional Clinical Doctorate & $5,5.2 \%$ \\
\hline & Academic Doctorate & $17,17.7 \%$ \\
\hline
\end{tabular}

\section{Data collection}

An email was sent to the heads of athletic training departments at the universities and colleges in the United States, asking them to forward the email to their athletic training staff. The same email was also sent to program directors of accredited athletic training programs and the members of local athletic training organizations. The message was then forwarded to all prospective participants inviting them to participate in an electronic survey via a hyperlink from a web-based server (Qualtrics Inc., Provo, UT) in July and August 2020. The inviting message contained information about the researchers, the purpose of the study, the nature of the survey, and assurances that the participants could opt to not complete the survey at any time. A follow-up email was sent a week after the initial email, and the survey was left open for an additional week prior to the survey being closed for statistical analysis.

\section{Instrument}

After the informed consent and demographics section, the survey included questions addressing formal education and training regarding cupping therapy, as well as the frequency of usage for cupping therapy during typical clinical practice. Participants were also asked to provide information about the equipment they used to apply cupping therapy.
To create the questions assessing actual knowledge of cupping therapy, a review of recent literature of published studies and textbooks was performed to inform the content [5-9]. This content was designed to be similar to questions given to athletic training students in an accredited athletic training course. These questions were developed by a researcher with content expertise and reviewed by a researcher with experience instructing pharmacology courses for athletic trainers.

Ultimately, the survey consisted of 35 questions. These questions included: one question regarding informed consent, four multiple choice and two fill in the blank questions on demographics, seven multiple choice and one multiple answer question on education and current usage of over the counter medications, 10 Likert-scale items that assessed participants' perceived knowledge, and 10 multiple choice and two multiple answer questions that evaluated participants' actual knowledge.

\section{Statistical analysis}

Data from participant responses was downloaded and analyzed using a commercially-available statistics package (SPSS Version 26, IBM, Armonk, NY). 79 completed responses were included in the data analysis. Measures of central tendency (means, standard deviations, frequencies) were calculated where appropriate. The 
researchers reverse scored four items from the perceived knowledge portion of the questionnaire and calculated a mean score for the 10 items. Additionally, the researchers calculated a total correct score per participant on the actual knowledge assessment. A Pearson correlation was calculated for the perceived knowledge mean score and the actual knowledge total score to identify a knowledge gap between what participants believed the knew and what they actually knew. Significance was set at $\mathrm{P}<.05$ a priori.

Results

\section{Education}

The majority of athletic trainers stated that they had attended a continuing education presentation on over the counter medication within the past two years $(57.3, \mathrm{n} \%=55)$. However, only $38.5 \%$ of athletic trainers reported attending a continuing education presentation on over the counter NSAIDs within the past two years (n = 37).

Table 2: Perceived Knowledge assessment most common response.

\begin{tabular}{|c|c|}
\hline Item & Most Common Response \\
\hline I could explain the benefits of over the counter NSAIDs to a patient & Agree $(n=43)$ \\
\hline I could assess a patient for indications, contraindications, and precautions for over the counter NSAIDs & Agree $(n=46)$ \\
\hline I could explain the physiological effects and theoretical foundations of over the counter NSAIDs & Agree $(n=34)$ \\
\hline I could describe the expected outcomes and adverse reactions of an over the counter NSAID & Agree $(n=42)$ \\
\hline I could select the proper over the counter dose for an NSAID & Strongly Agree $(n=40)$ \\
\hline I could explain the difference between ibuprofen and acetaminophen & Strongly Agree $(n=50)$ \\
\hline I could explain the sensations a patient should experience when taking an over the counter NSAID & Agree $(n=37)$ \\
\hline I do not feel very knowledgeable about over the counter NSAIDs & Disagree $(n=42)$ \\
\hline $\begin{array}{c}\text { I am skilled and proficient in the appropriate administration of over the counter NSAIDs based off my state } \\
\text { practice act }\end{array}$ & Agree $(n=43)$ \\
\hline I am likely to pursue continuing education to improve my knowledge and skills related to over the counter NSAIDs & Somewhat Agree $(n=30)$ \\
\hline
\end{tabular}

\section{Correlation between perceived and actual knowledge}

We identified a weak positive relationship between perceived knowledge mean scores and actual knowledge total correct sum scores $(\mathrm{r}=0.241, \mathrm{P}=.018)$. This suggested that as perceived knowledge of cupping therapy increased, actual knowledge of cupping therapy increased as well. However, actual knowledge was $7.44 \pm 1.68$ out of 10 . This indicated that actual knowledge of over the counter NSAIDs was lower than $75 \%$.

\section{Discussion}

The purpose of this study was to assess the perceived and actual knowledge of over the counter NSAIDs among credentialled athletic trainer. A secondary purpose was to determine the frequency of usage of over the counter NSAIDs among these athletic trainers. Our findings suggest that $75 \%$ of the athletic trainers surveyed use over the counter NSAIDs during a typical in their athletic training practice $(n=72)$. This frequency of usage mirrored athletic trainers' administrations of over the counter medications in general. These findings indicate that athletic trainers are frequently administering

\section{Clinical usage}

The majority of athletic trainers reported administering over the counter medications to patients at least once a week $(75.0 \%$, $\mathrm{n}$ = 72). These numbers mirrored athletic trainers' reported administration of over the counter NSAIDS at least once a week $(75.0 \%, \mathrm{n}=72)$. The most common medications kept in athletic training facilities were ibuprofen $(95.8 \%, \mathrm{n}=92)$, acetaminophen $(94.8 \%, \mathrm{n}=91)$, naproxen $(59.4 \%, 57)$, and diphenhydramine $(58.3 \%, \mathrm{n}=56)$. The least common medications kept in athletic training facilities were cetirizine hydrochloride $(14.6 \%, \mathrm{n}=14)$, aspirin $(26.0 \%, n=25)$, and dextromethorphan $(26.0 \%, n=25)$.

\section{Perceived knowledge}

The participants had a mean sum of the perceived knowledge items ( $\mathrm{n}=10$ ) of $5.77 \pm 0.81$ out of 7 . This finding suggested that most participants were above mid-range of perceived knowledge of over the counter NSAIDs. Frequency count of the most common correct responses are provided in Table 2. over the counter NSAIDs and are using them with a similar frequency to other over the counter medications.

Compared to previous studies on athletic training skills, athletic trainers demonstrated a similar lack of knowledge retention [1012]. This suggests there are many tasks and skills that are susceptible to knowledge deterioration in the athletic training profession. Additionally, there was a weak positive relationship observed between perceived knowledge and actual knowledge of over the counter NSAIDs in this study. Even with this positive relationship, the average actual knowledge score among participants was lower than $75 \%$. Given that the majority of participants reported not attending a continuing education presentation on over the counter NSAIDs within the past two years, these findings suggest a need for emphasizing education on over the counter NSAIDs within athletic training curriculum and continuing education programming.

Previous studies have shown that clinical athletic training skills are subject to deterioration if they are not practiced over time [10-12]. Indeed, some studies have suggested that skills such as cardiopulmonary resuscitation can decrease in as little as six 
months [13]. Athletic trainers are more likely to administer over the counter NSAIDs on a weekly basis compared to cardiopulmonary resuscitation. Still, there is a potential for knowledge and skill deterioration if the modality is not used regularly. In the absence of clinical practice, continuing education has been shown to be a valuable method of knowledge transfer and retention $[11,12]$.

These findings may suggest a need to include more extensive information about the theory and concepts related to over the counter NSAIDs within athletic training curriculums. In the event that this proves difficult for athletic training education programs, continuing education programming should be considered for knowledge transfer of the definition, modes of action, indications, and contraindications of over the counter NSAIDs. When these continuing education presentations are created, they should be assessed and reassessed to make sure that they are effective and efficient at improving knowledge of over the counter NSAIDs among athletic trainers.

A possible limitation of this study was the number of participants. However, this is a similar limitation that other surveybased studies on athletic trainers have dealt with. That being said, this limitation may affect the generalizability of the results when looking to analyze across the profession [11,12]. Additionally, the response and completion rate was higher than some survey-based studies on athletic trainers $[11,12]$.

The findings of this study support those of previous research that suggest a need for further research on the perceived knowledge of clinical skills and tasks among athletic trainers. To the authors' knowledge, the first study to examine the perceived and actual knowledge of over the counter NSAIDs among athletic trainers. Future research should also be directed towards knowledge transfer and retention related to over the counter NSAIDs and other commonly administered over the counter medications.

\section{Conclusion}

In conclusion, over the counter NSAIDs are frequently administered by athletic trainers. However, athletic trainers averaged lower than $75 \%$ when actual knowledge of over the counter NSAIDs was assessed. This demonstrates the need for education on over the counter NSAIDs that are frequently used by athletic trainers. Given that continuing education has been suggested as a means of knowledge acquisition and retention, clinicians might benefit from creation of more extensive continuing education programming on over the counter NSAIDs.

\section{References}

1. Babarinde O, Ismail H, Schellack N (2018) An overview of the management of muscle pain and injuries. Professional Nursing Today 22(1): 14-23.

2. Green T, Wilson G, Martin D, Fallon K (2019) What is the quality of clinical practice guidelines for the treatment of acute lateral ankle ligament sprains in adults? A systematic review. BMC Musculoskeletal Disorders 20: 394.

3. Gorski T, Cadore EL, Pinto SS, da Silva EM, Correa CS, et al. (2011) Use of nonsteroidal anti-inflammatory drugs (NSAIDs) in triathletes: Prevalence, level of awareness, and reasons for use. Br J Sports Med 45(2): 85-90.

4. Alaranta A, Alaranta H, Helenius I (2008) Use of prescription drugs in athletes. Sports Med 38(6): 449-463.

5. Chang CJ, Weston T, Higg JD, Ohkubo M, Sauls A, et al. (2018) Interassociation consensus statement: The management of medications by the sports medicine team. Journal of Athletic Training 53(11): 11031112.

6. Wolf DA, Miller TW, Pescatello LS, Barnes C (2011) National Collegiate Athletic Association Division I athletes' use of nonprescription medication. Sports Health 3(1): 25-28.

7. Bates DK (2020) Opioids and an active population: Athletic trainers' perceptions. The Internet Journal of Allied Health Sciences and Practice 18(1).

8. Da Silva E, Pinto RS, Cadore EL, Kruel LF (2015) Nonsteroidal antiinflammatory drug use and endurance during running in male longdistance runners. Journal of Athletic Training 50(3): 295-302.

9. Houglum JE, Harrelson GL, Seefeldt TM (2016) Principles of pharmacology for athletic trainers. SLACK Incorporated, Thorofare, New Jersey, USA, 2016.

10. Cage SA, Warner BJ, Gallegos DM, Winkelmann ZK (2020) Athletic trainers perceived and actual knowledge of cupping therapy concepts. Journal of Sports Medicine and Allied Health Sciences 5(3).

11. Cage SA, Winkelmann ZK, Warner BJ, Gallegos DM (2020) Perceived and actual knowledge of cupping therapy concepts among athletic training preceptors in CAATE accredited programs. Research \& Investigations in Sports Medicine 6(3): 514-518.

12. Schellhase K, Plant J, Rothschild C (2015) Collegiate athletic trainers' perceived and actual knowledge of therapeutic ultrasound concepts. International Journal of Athletic Therapy and Training 20(5): 43-53.

13. Yang CW, Yen ZS, McGowan JE, Chen HC, Chiang WC, et al (2012) A systematic review of retention of adult advanced life support knowledge and skills in heathcare providers. Resuscitation 83(9): 1055-1060. 